\title{
Cultural Perspective-based Chinese Adolescent Martial Arts Teaching Development Research
}

\author{
Xiaobin $\mathrm{Xu}^{*}$ \\ Zhejiang Yuying College of Vocational Technology, Hangzhou 310018, ZheJiang, China
}

\begin{abstract}
In contemporary China, martial arts teaching is an important mean to let Chinese martial arts culture to be inherited and developed, but with the diversification of teaching courses, it lets Chinese martial arts teaching's position to be worsen in the mind of adolescent, more and more special courses similar to martial arts are replaced by literacy class. The paper studies Chinese martial arts teaching development conditions in contemporary social environment from the perspective of adolescent martial arts teaching. First, it makes statistics of Chinese schools' teachers and students martial arts culture cognition degree, and analyses their satisfaction degree, and then learns martial arts culture popularization among school teaching; secondly, utilize correlation analysis method and adaptive filtering method, it analyses Chinese adolescent favourite martial arts events. And then further get the conclusion: affected by martial arts events technical content, adolescents prefer to young Changquan, Taijiquan and others martial arts courses of lower technical content requirement and field constraint, and their satisfaction degree is higher. To let Chinese martial arts culture to be continued to carry forward and inherit, it should enlarge teachers and students cognition to martial arts culture and emphasis on.
\end{abstract}

Keywords: Adolescent sports, martial arts culture, martial arts teaching, statistical analysis, traditional sports.

\section{INTRODUCTION}

Adolescent is related to lifeblood of a country's future, in order to develop Chinese martial arts culture, enhance adolescent understanding, improve adolescent physical quality, let adolescent to focus on Chinese excellent culture from childhood, and let adolescent to be all-round developed in morality, intelligence, physique, aesthetics and labor, it should try to penetrate martial arts teaching into school as earlier as possible [1].

Wang Yi-Ding in the article "Changchun city primary school martial arts teaching developing conditions research", by taking Chinese Changchun city primary school martial arts teaching as an example, he made research on martial arts teaching, pointed out that it must change current stage primary school martial arts teaching conditions, government should increase emphasis and investment on it, and take excellent faculty as firm backup force guarantee [2], should research on teaching methods, find out methods to adapt to adolescent martial arts teaching, and then increase adolescent interests, and meanwhile let them to pass down these excellent cultures [3]. Chen Jian-Dong in "Yangpu district primary school martial arts current conditions and counter measures study", according to researches on Yangpu district adolescent martial arts teaching, finally he pointed out that nation should formulate and revise martial arts teaching courses to fit for adolescent learning, not only should strengthen in physique but also intensify in the theoretical knowledge [4], secondly it should cultivate a group of teachers with professional martial arts knowledge, in this way simultaneous intensified hardware and books so that let Chinese adolescent teaching to be improved and let adolescent martial arts to be further developed [5]. Sun Bei-Bei in "Henan province vocational colleges' martial arts teaching status and counter measures study", took Henan province university students as research objects, the thesis put forward that Chinese equipments and facilities, martial arts atmosphere [6], contents dullness, shortage in faculty these factors all affected Chinese adolescent martial arts teaching quality, which provided significant references for improving Chinese martial arts teaching, and meanwhile provided guarantee for enhancing adolescent physique [7].

The paper through referencing multiple scholars research results, utilizes mathematical methods to make quantitative analysis of Chinese adolescent martial arts teaching influence factors, and combines with contemporary Chinese national conditions analysis upmost factors, by visiting multiple martial arts professional coaches and teaching research scholars, makes comprehensive analysis and puts forward teaching ways and methods of Chinese characteristics, which offers help to clear away obstacles that affect adolescent martial arts teaching development [8].

\section{CONTEMPORARY ADOLESCENT TEACHING DEVELOPMENT INFLUENCE FACTORS}

In current stage of China, adolescent powerfulness is an important guarantee to future China in international society competition, only build adolescent to be $21^{\text {st }}$ century youth with four haves then can make contributions to Chinese future development. 
Table 1. Primary school knowledge about martial arts.

\begin{tabular}{|c|c|c|c|c|c|}
\hline Primary school & Know Very Well & Know & General & Don't Understand & Know Nothing About it \\
\hline \hline Number of people & 12 & 25 & 37 & 18 & 8 \\
\hline Proportion (\%) & $12 \%$ & $25 \%$ & $37 \%$ & $18 \%$ & $8 \%$ \\
\hline
\end{tabular}

Table 2. Secondary school knowledge about martial arts.

\begin{tabular}{|c|c|c|c|c|c|}
\hline Secondary School & Know Very Well & Know & General & Don't Understand & Know Nothing About it \\
\hline \hline Number of people & 15 & 34 & 35 & 11 & 5 \\
\hline Proportion (\%) & $15 \%$ & $34 \%$ & $35 \%$ & $11 \%$ & $5 \%$ \\
\hline
\end{tabular}

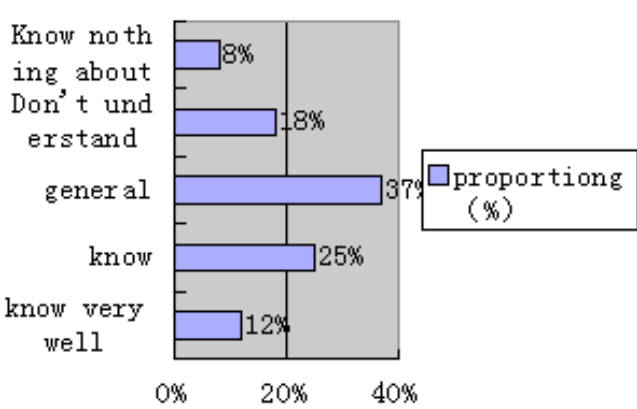

Fig. (1). Know the situation of primary and secondary schools of martial arts.

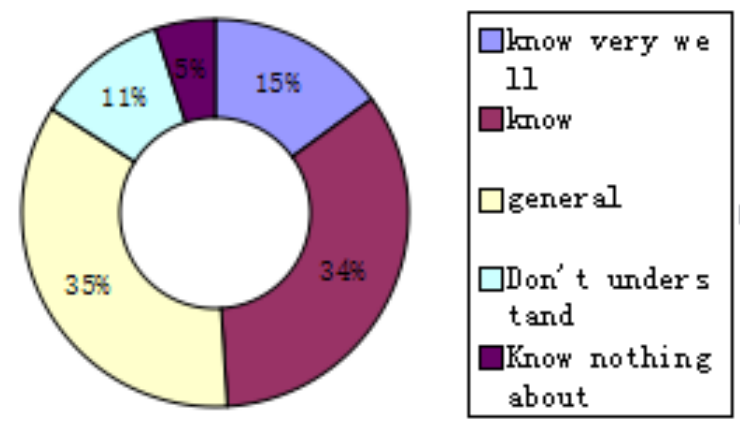

Fig. (2). Know the situation of primary and secondary schools of martial arts.

\subsection{Student's Knowledge About Martial Arts}

Martial arts culture is extensive and profound, only let adolescent to understand and be interested then can let adolescent to carry forward it. Below Tables $\mathbf{1}$ and $\mathbf{2}$ are adolescent group's secondary school and primary school's martial arts knowledge status table, data is from internet statistical data (Figs. 1 and 2).

According to above two secondary school and primary school pie chart 1-2, it is clear that among secondary school and primary school's students, considerable parts of people knowledge and feeling about martial arts are general, adolescent that know it very well are little that is because school publicity on martial arts is not enough, it reflects Chinese teaching's martial arts culture is to be further strengthened.

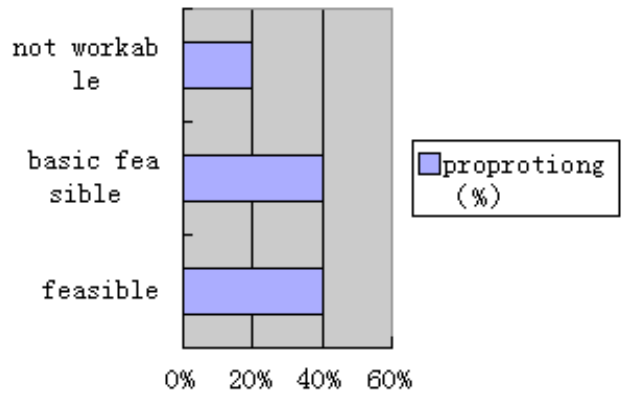

Fig. (3). Teacher and student questionnaire evaluation results TAB.

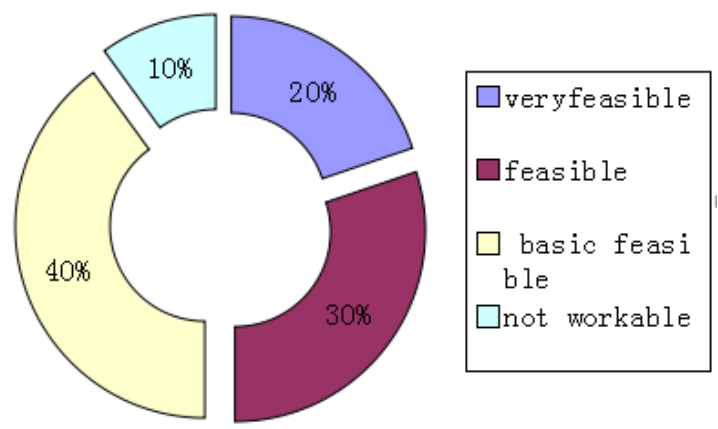

Fig. (4). Teacher and student questionnaire evaluation results TAB.

\subsection{Students Evaluation Status on Martial Arts}

Different places' schools, teachers and students' evaluation on contemporary martial arts teaching is different, below Tables $\mathbf{3}$ and $\mathbf{4}$ are teachers and students to martial arts teaching evaluation tables.

From above statistical (Figs. 3 and 4), it is clear that among teachers, above $40 \%$ people think that martial arts teaching is feasible, which proves teachers recognition degree on martial arts is higher that provides faculty for martial arts teaching development; in students evaluation, nearly $60 \%$ students suppose it feasible that provides guarantee for Chinese martial arts cultural development.

\subsection{Chinese Secondary and Primary Schools' Martial Arts Teaching Objectives Positioning Conditions}

In Chinese social life, due to government department, associations and communities as well as social sponsor organizations lay emphasis on adolescent physical health, 
Table 3. Teachers and students questionnaire e valuation result statistical table.

\begin{tabular}{|c|c|c|c|}
\hline Teachers Evaluation & Feasible & Basic Feasible & Not Workable \\
\hline \hline Number of people & 4 & 4 & 2 \\
\hline Proportion(\%) & $40 \%$ & $40 \%$ & $20 \%$ \\
\hline
\end{tabular}

Table 4. Teachers and students questionnaire evaluation result statistical table.

\begin{tabular}{|c|c|c|c|c|}
\hline Students Evaluation & Very Feasible & Feasible & Basic Feasible & Not Workable \\
\hline \hline Number of people & 2 & 3 & 4 & 1 \\
\hline Proportion (\%) & $20 \%$ & $30 \%$ & $40 \%$ & $10 \%$ \\
\hline
\end{tabular}

Table 5. Chinese secondary and primary schools' martial arts teaching objective positioning conditions table.

\begin{tabular}{|c|c|c|c|c|c|}
\hline Teaching Objective & Sports Participation & Sports Skills & Physical Health & Psychological Health & Social Adaptation \\
\hline \hline Amount of schools & 9 & 11 & 8 & 6 & 4 \\
\hline $\begin{array}{c}\text { Selection frequency } \\
\text { (\%) }\end{array}$ & $75 \%$ & $91.7 \%$ & $66.7 \%$ & $50 \%$ & $33.3 \%$ \\
\hline Ranking & 2 & 1 & 3 & 4 & 5 \\
\hline
\end{tabular}

Table 6. Chinese secondary and primary schools' martial arts teaching objective positioning conditions table.

\begin{tabular}{|c|c|c|c|}
\hline & Senior High School & Junior High School & Primary School \\
\hline \hline Amount of schools & 2 & 5 & 3 \\
\hline Selection frequency (\%) & $20 \%$ & $50 \%$ & $30 \%$ \\
\hline Ranking & 3 & 1 & 2 \\
\hline
\end{tabular}

and meanwhile increase physical quality cultivation in teaching, below Tables $\mathbf{5}$ and $\mathbf{6}$ are secondary and primary schools' teaching objective conditions tables.

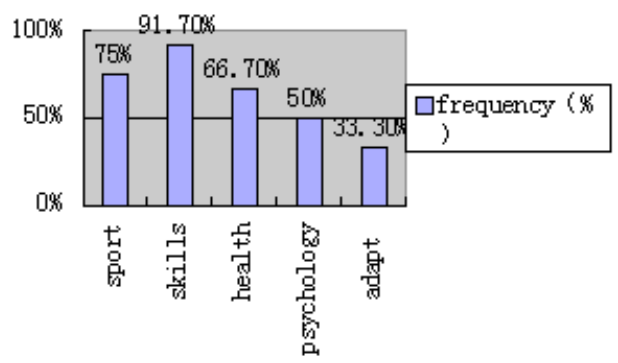

Fig. (5). Martial arts teaching of primary and secondary schools.

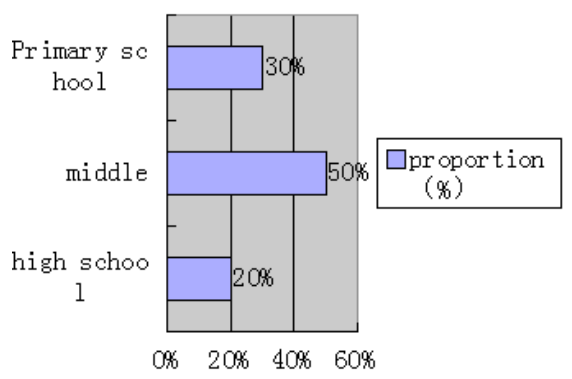

Fig. (6). Martial arts teaching of primary and secondary schools.
From above (Figs. 5 and 6) Chinese several secondary and primary schools teaching objective setting conditions investigation table, it is clear that sports skills intention has arrived at satisfaction degree in many schools, and social adaptation degree in teaching only reaches the goal in little schools, which shows that school martial arts teaching should strengthen balanced development of all aspects.

\section{ADOLESCENT COGNITION DEGREES ON MAR- TIAL ARTS TEACHING}

\subsection{Correlation Analysis Guiding Thought}

Common correlation coefficient has Pearson correlation coefficient and Spearman rank correlation coefficient. The paper utilizes Pearson correlation coefficient to make correlation comparison.

Pearson correlation coefficient is used to show two variables similarity extent mathematical statistical quantity, it can be used to make quantitative calculation on two variables similarities. Its calculation formula is as following:

$$
\begin{aligned}
& \rho(X, Y)=\frac{\operatorname{cov}(X, Y)}{\sigma_{x} \sigma_{y}}=\frac{E\left(\left(X-\mu_{x}\right)\left(Y-\mu_{y}\right)\right)}{\sigma_{x} \sigma_{y}} \\
& \text { And } \mu_{x}=E(X), \sigma_{X}{ }^{2}=E\left(X-\mu_{x}\right)^{2}=E\left(X^{2}\right)-E^{2}(X)
\end{aligned}
$$


Table 7. Each kind of martial arts penetrating into fitness.

\begin{tabular}{|c|c|c|c|c|c|}
\hline & Instrument & Young Changquan & Catch and Grapple & Striker & Women's Self Defense \\
\hline \hline Sports attitudes $R$ & $.326^{* *}$ & $.119^{* *}$ & $.495^{* *}$ & $.148^{* *}$ & $.122^{*}$ \\
\hline$P$ & .004 & .001 & .002 & .008 & .036 \\
\hline
\end{tabular}

*It represents to arrive at significant correlation in the level 0.05 .

**It represents to arrive at significant correlation in the level 0.01 .

Table 8. Adolescent martial arts practice duration correlation analysis data table.

\begin{tabular}{|c|c|c|c|c|c|}
\hline & Instrument & Young Changquan & Catch and Grapple & Striker & Women's Self Defense \\
\hline \hline Sports attitudes $R$ & $.225^{* *}$ & $.395^{* *}$ & $.341^{* *}$ & $.313^{* *}$ & $.118^{*}$ \\
\hline$P$ & .002 & .013 & .006 & .001 & .043 \\
\hline
\end{tabular}

*It represents to arrive at significant correlation in the level 0.05 .

** It represents to arrive at significant correlation in the level 0.01 .

Therefore, Pearson correlation coefficient can also be written as: $\rho(X, Y)=\frac{E(X Y)-E(X) E(Y)}{\sqrt{E\left(X^{2}\right)-E^{2}(X)} \sqrt{E\left(Y^{2}\right)-E^{2}(Y)}}$

When two variables Pearson correlation coefficient gets closer to 1 or -1 , it shows the two correlation is big, or closely related. It gets closer to 1 shows the two are in positive correlation, on the contrary it gets closer to -1 shows the two are in negative correlation.

\subsection{Adolescent Sports Information Receiving and Sports Attitudes Correlation Analysis}

Make correlation analysis of adolescent martial arts events selection data table and attitudes data table, according to above process, utilize spss software; it gets following Tables $\mathbf{7}$ and $\mathbf{8}$ results.

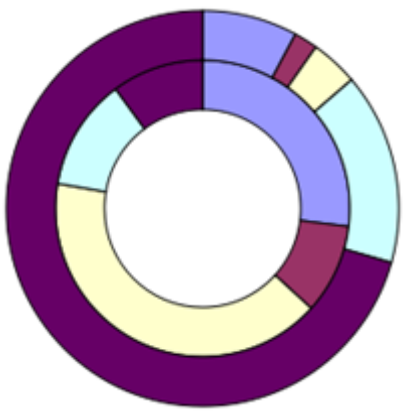

$\square$ Instrument

Young chang chuan

$\square$ Catch and grapple

$\square$ Striker

口Women's Self Defense

Fig. (7). The relationship.

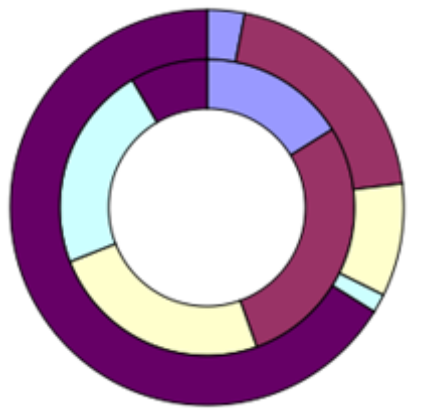

$\square$ Instrument

$\square$ Young chang chuan

$\square$ Catch and grapple

$\square$ Striker

$\square$ Women's Self Defense

Fig. (8). The relationship.

By above two (Figs. $\mathbf{7}$ and $\mathbf{8}$ ), it is clear that in secondary and primary schools' martial arts teaching, the events of most easily practicing and highest students satisfaction degree are instrument martial arts, Taijiquan and young changquan, because these teaching events are lower in the demands of fields selection and technical requirement, which also conforms to contemporary China social development trends and situations, it provides extremely precious references for Chinese martial arts teaching should increase investment on other events.

\subsection{Adolescent Satisfaction Degree on Martial Arts Dif- ferent Events}

Martial arts events that penetrate into adolescent teaching and fitness aspects are quite a lot that respectively have instrument martial arts, young changquan, catch and grapple, striker, Wing Chun, Tai Chi, women's self defense and others, below Table 9 is statistical table about relevant martial arts events satisfaction degrees in the mind of adolescent.

In above several kinds of martial arts teaching cognition degrees, young changquan, instrument martial arts and Taijiquan these three items are of adolescent most satisfaction, in adolescent cognition to five-step boxing, Wing Chun and others, due to technical requirement is higher, satisfaction degrees among students are little, and nation should advertise different martial arts events to arrive at balanced development.

\section{ADAPTIVE FILTERING METHOD-BASED CHI- NESE MARTIAL ARTS TEACHING INVESTMENT RESEARCH}

\subsection{Guiding Thought of Adaptive Filtering Method}

Similar to moving average method and exponential smoothing method, adaptive filtering method is on the basis of historical observation value with time developing and changing, do weighted average on it and then go ahead with prediction. Adaptive filtering method should define optimal weight that has smallest errors. The method is utilizing a group of given historical data to calculate corresponding 
Table 9. Correlation analysis data table.

\begin{tabular}{|c|c|c|c|c|c|c|}
\hline & $\begin{array}{c}\text { Martial Arts In- } \\
\text { strument }\end{array}$ & Young Changquan & Catch and Grapple & Striker & $\begin{array}{c}\text { Women's Self } \\
\text { Defense }\end{array}$ & \begin{tabular}{c} 
Taijiquan \\
\hline \hline Satisfaction attitude $R$
\end{tabular} \\
\hline$P$ & $.175^{* *}$ & $.116^{* *}$ & $.121^{* *}$ & $.079^{* *}$ & $-.061^{*}$ & $-.116^{* *}$ \\
\hline & .000 & .000 & .001 & .002 & .015 & .001 \\
\hline Satisfaction attitude $R$ & Five-step boxing & Eight section brocade & Nunchaku & Wing Chun & Sword dance & Else \\
\hline$P$ & -.045 & -.057 & $-.069^{*}$ & -.054 &. $.105^{* *}$ & $.0009^{* *}$ \\
\hline
\end{tabular}

*It represents to arrive at significant correlation in the level $0.05 ;^{* *}$ It represents to arrive at significant correlation in the level 0.01 .

predicted value, and calculate errors, according to calculated errors, it adjust pre-defined weights, so that let predicted value error to be reduced to the lowest. In general, once calculation cannot get optimal weight, so we need to repeatedly predict, by constantly calculating, adjusting defined weights, then get a group of weight when predicted value error being the lowest that is solved optimal weight.

Adaptive filtering method basic prediction formula is:

$\hat{y}_{t+1}=\omega_{1} y_{t}+\omega_{2} y_{t-1}+\cdots+\omega_{N} y_{t-N+1}=\sum_{i=1}^{N} \omega_{i} y_{t-i+1}$

In formula, $y_{t+1}$ is the $t+1$ predicted value, $\omega_{i}$ is the $t-i+1$ observation weight, $y_{t-i+1}$ is the $t-i+1$ observation value, $N$ is the number of weights. Below is formula of weight adjustment:

$\omega_{i}^{\prime}=\omega_{i}+2 k \bullet e_{i+1} y_{t-i+1}$

Among them, $i=1,2, \ldots, N, t=N, N+1, \ldots, n, n$ is the number of sequence data, $\omega_{i}$ is the $i$ weight before adjusting, $\omega_{i}^{\prime}$ is the $i$ weight after adjusting, $k$ is learning constant, $e_{i+1}$ is the $t+1$ predicted error.

Above formula shows after adjustment weight is equal to the sum of original adjustment weight and error adjustment item, and the adjustment item mainly includes prediction error, original historical observation value, learning constant.

\subsection{Adaptive Filtering Method Data Processing}

Here, $N=2$. Take initial weight $\omega_{1}=0.97, \omega_{2}=0.96$, and set $k=0.9, t$ value starts from $N=2$, that when $t=2$, According to prediction formula, solve prediction value when $t+1=3$ :

$$
\hat{y}=\hat{y_{3}}=\omega_{1} y_{2}+\omega_{2} y_{1}=588.24
$$

Calculate predicted errors: $e_{t+1}=e_{3}=y_{3}-\hat{y}_{3}=2.78$

$$
\omega_{i}^{\prime}=\omega_{i}+2 k \bullet e_{i+1} y_{t-i+1}
$$

Calculate: $\omega_{1}^{\prime}=\omega_{1}+2 k \bullet e_{3} y_{2}=0.975$

$\omega_{2}^{\prime}=\omega_{2}+2 k \bullet e_{3} y_{1}=0.964$

Then $t+1$ and repeat above steps. When $t=3$ :

Utilize obtained weight, calculate predicted value when $t+1=4$ that is to take one headmost observation value $y_{1}$, add a new observation value ${ }^{y_{3}}$. Then:

$\hat{y}_{t+1}=\hat{y}_{4}=\omega_{1}^{\prime} y_{3}+\omega_{2}^{\prime} y_{2}=881.81$

Calculate prediction error:

$e_{t+1}=e_{4}=\hat{y}_{4}-\hat{y}_{4}=1.05$

Adjust weight:

$\omega_{1}^{\prime}=\omega_{1}+2 k \bullet e_{4} y_{4}=0.989$

$\omega_{2}^{\prime}=\omega_{2}+2 k \bullet e_{4} y_{3}=-0.02$

When $t=5$,

Utilize obtained weight, calculate predicted value when $t+1=6$ :

$\hat{y}_{t+1}=\hat{y}_{4}=\omega_{1}^{\prime} y_{4}+\omega_{2}^{\prime} y_{3}=880.56$

Calculate prediction error:

$e_{t+1}=e_{5}=\hat{y}_{5}-\hat{y}_{5}=8.45$

Weight:

$$
\begin{aligned}
& \omega_{1}^{\prime}=\omega_{1}+2 k \bullet e_{5} y_{4}=0.9892 \\
& \omega_{2}^{\prime}=\omega_{2}+2 k \bullet e_{3} y_{3}=-0.0251
\end{aligned}
$$

And then, by three times adjusting weights, finally it gets optimal weight group is

$$
\omega_{1}^{\prime}=0.9892, \omega_{2}^{\prime}=-0.0251
$$

Draw above predicted Chinese martial arts education investment into following broken line figure, better analyze martial arts education investment trend: 
Table 10. National martial arts teaching investment.

\begin{tabular}{|l|c|c|c|c|c|}
\hline \multirow{2}{*}{ National martial arts teaching investment sum (one hundred million Yuan) } & 2008 & 2009 & 2010 & 2011 & 2012 \\
\cline { 2 - 7 } & 3.5 & 3.3 & 5.1 & 5.2 \\
\hline
\end{tabular}

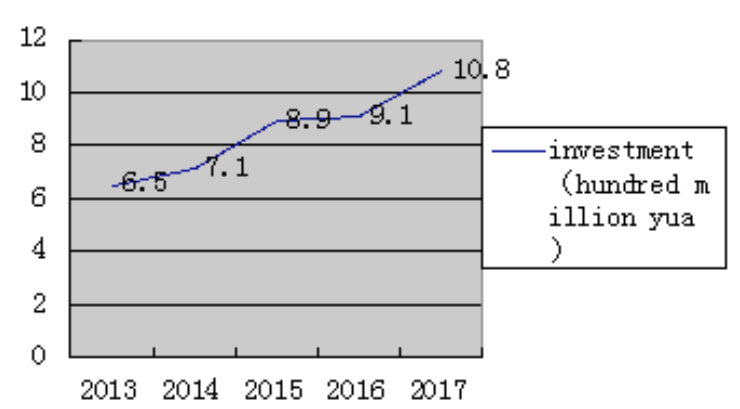

Fig. (9). Martial arts education investment from 2013 to 2017.

From above broken line Fig. (9), it is clear that Chinese investment on school martial arts teaching is further increased with previous statistical data, only then can let Chinese martial arts education to be normal and stable developed, only then can let adolescent to understand martial arts and carry forward Chinese martial arts culture Table $\mathbf{1 0 .}$

\section{CONCLUSION}

The paper through analyzing contemporary martial arts teaching status, it gets conclusion: Most of Chinese adolescents and teachers are satisfied with martial arts teaching, but people know martial arts teaching very well are little, and due to causes of high technical requirement, martial arts haven't been universally recognized, and China must strengthen investment in martial arts teaching aspect. Secondly, the paper through correlation analysis and filtering method, carries out comprehensive analysis of Chinese martial arts education development influence factors, and then gets that teachers professional standards are main factors that affect martial arts development, and in martial arts teaching, young changquan, Taijiquan and instrument martial arts are contemporary adolescent relative preferred martial arts events that is because with respect to other martial arts events, these three martial arts events demands in fields and teachers' technical standards aspects are relative lower and easier to popularize and develop. Finally, combine with above analysis, it conceives Chinese martial arts teaching development orientations and future development modes, and puts forward problems that present Chinese martial arts teaching comes across, and relevant solution, which provides precious opinions for Chinese martial arts teaching.

\section{CONFLICT OF INTEREST}

The author confirms that this article content has no conflict of interest.

\section{ACKNOWLEDGEMENTS}

Declared none.

\section{REFERENCES}

[1] J. Chen, and Y. Shi, "A countermeasure study about realizing sustainable development of basketball professionalism in China," $J$. Beijing Sport Univ., vol. 25, no. 3, pp. 301-302, 2002.

[2] S-F. Huang, "Research on the countermeasures for chinese man's basketball team in london olympics," Chin. Sport Sci. Technol., vol. 47 , no. 1 , pp. 12-20, 2011.

[3] N. Jia, and H.C. Sun, "Objectives of developing the Chinese competitive sports of the early $21^{\text {st }}$ century and studies of the countermeasures," J. Wuhan Inst. Phys. Educ., vol. 35, no. 6, pp. 1-4, 2001

[4] D-C. JIA, "Countermeasure and causation of imbalance of teams athletic competence in CBA," J. Hebei Inst. Phys. Educ., vol. 23, no. $1,2009$.

[5] M.A. Jinrong, and S. Gong, "Some problems of WCBA league teams and foreign main centre," J. Shenyang Sport Univ., vol. 31, no. 3, pp. 84-88, 2012

[6] Y. Wang, and Y. Cheg, "Strength pattern of current world man's basketball from the view of $15^{\text {th }}$ world man's basketball championships," Chin. Sport Sci. Technol., vol. 43, no. 4, pp. 77-81, 2007.

[7] B. Xu, and T. Xie, "A study on the landslide of china's competitive basketball games and countermeasures," J. Beijing Sport Univ., vol. 5, no. 5, pp. 101-105, 2010.

[8] H. Xue, "A study of the causes and countermeasures of the decline of competitive basketball in China," Bull. Sport Sci. Technol., vol. 21, no. 2, pp. 26-27, 2013.

(C) Xiaobin Xu; Licensee Bentham Open.

This is an open access article licensed under the terms of the (https://creativecommons.org/licenses/by/4.0/legalcode), which permits unrestricted, noncommercial use, distribution and reproduction in any medium, provided the work is properly cited. 